\title{
O PAPEL DA ECOENDOSCOPIA NO DIAGNÓSTICO DAS NEOPLASIAS CÍSTICAS PRIMÁRIAS DO PÂNCREAS*
}

\author{
Simone Guaraldi ${ }^{1}$, Evandro Sá ${ }^{2}$, Sérgio Romano ${ }^{3}$, Antonio Carlos Pires Carvalho $^{4}$
}

Resumo 0 diagnóstico das lesões císticas pancreáticas pelos métodos de imagem, especialmente as de pequeno tamanho, é cada vez mais freqüente. Em alguns casos, ele representa um dilema em relação à terapêutica, podendo ser pseudocistos inflamatórios, neoplasias primárias ou secundárias. Para a decisão terapêutica, é necessário definir se a neoplasia é benigna, maligna ou potencialmente maligna. Hoje, a ecoendoscopia é considerada o exame padrão-ouro para a investigação do pâncreas, fornecendo dados sobre a morfologia destas lesões e possibilitando, por meio da punção guiada em tempo real, a colheita de material para avaliação citológica e dos marcadores tumorais. Este procedimento é considerado seguro e eficiente e apresenta taxas de sensibilidade e especificidade altas e de morbidade e de complicações baixas. No diagnóstico das lesões mucinosas do pâncreas, os fatores preditivos mais significativos para o diagnóstico diferencial são a presença de septos, os nódulos murais e as alterações parenquimatosas, para o qual as taxas de sensibilidade, especificidade e grau de exatidão são, respectivamente, $94 \%, 85 \%$ e $88 \%$. Os autores têm por objetivo revisar as principais neoplasias císticas primárias do pâncreas, enfatizando a aplicação da ecoendoscopia no diagnóstico definitivo dessas neoplasias.

Unitermos: Ecoendoscopia; Ultra-sonografia endoscópica; Endossonografia; Neoplasia cística do pâncreas.

Abstract Usefulness of echoendoscopy in the diagnosis of primary cystic neoplasms of the pancreas

Pancreatic cystic lesions, particularly small lesions, are more easily diagnosed nowadays with the use of imaging methods. In some cases, the diagnosis represents a challenge to establish the treatment, as it can range from inflammatory pseudocysts to primary or metastatic cystic neoplasms. In order to choose the treatment, it is necessary to determine if the lesion is benign, borderline, or malignant. Currently, echoendoscopy is considered the gold standard procedure for pancreatic evaluation as it clearly shows the morphology of the lesion, and also allows the acquisition of pancreatic material for cytological and tumor markers studies using fine needle aspiration biopsy. This procedure is considered safe and efficient with high rates of sensibility and specificity and low rates of complications and morbidity. The presence of septa, mural nodules and irregularities in the parenchyma are the most significant predictive factors for the differential diagnosis of mucinous pancreatic-cystic lesions for which sensibility, specificity and accuracy rates are $94 \%, 85 \%$ and $88 \%$, respectively. The aim of the authors in this study is to review the major primarycystic-pancreatic neoplasms with emphasis in the application of echoendoscopy for the definite diagnosis of these lesions.

Key words: Echoendoscopy; Endoscopic ultrasound; Endosonography; Pancreatic cystic neoplasm.

\section{INTRODUÇÃO}

A descoberta de uma lesão cística do pâncreas pelos métodos de imagem, especialmente as de pequeno tamanho, é uma

* Trabalho realizado no Departamento de Radiologia da Faculdade de Medicina da Universidade Federal do Rio de Janeiro (UFRJ), Rio de Janeiro, RJ.

1. Mestre do Departamento de Radiologia da UFRJ, Membro Titular da Sociedade Brasileira de Endoscopia Digestiva - Sobed, "Attestation de Formation Spécialisée Approfondie" (A.F.S.A.) em Ecoendoscopia pela Faculdade de Medicina da Universidade do Mediterrâneo, França.

2. Membro Titular da Sobed, "Fellowship" em Endoscopia Digestiva pelo Keio Hospital, Japão.

3. Membro Titular da Sociedade Brasileira de Patologia.

4. Professor Adjunto-Doutor do Departamento de Radiologia da Faculdade de Medicina da UFRJ.

Endereço para correspondência: Dra. Simone Guaraldi. Rua Visconde de Pirajá, 259/702, Ipanema. Rio de Janeiro, RJ, 22410-001. E-mail: s.guaraldi@ecoendoscopia.com

Recebido para publicação em 30/7/2004. Aceito, após revisão, em 23/9/2004. situação cada vez mais frequiente na prática clínica, e representa, em alguns casos, um dilema em relação à conduta terapêutica a ser seguida.

As lesões císticas do pâncreas compreendem os pseudocistos inflamatórios e as neoplasias císticas em $80 \%$ a $90 \%$ e $10 \%$ a $15 \%$ dos casos, respectivamente ${ }^{(\mathbf{1 , 2})}$. As neoplasias císticas podem ser subdivididas em dois grupos de tumores: os císticos primários e os com degeneração cística secundária. No primeiro grupo estão as neoplasias epiteliais e incluem o cistadenoma seroso, o cistadenoma mucinoso, a neoplasia intraductal mucinosa papilífera, a neoplasia epitelial pseudopapilar sólida e as neoplasias mesenquimais (linfangiomas e teratomas). Os tumores com degeneração cística secundária incluem as neoplasias exócrinas pancreáticas sólidas (adenocarcinoma ductal) e as endócrinas (tumores das células das ilhotas). Outras lesões císticas do pâncreas incluem o cisto solitário, a policistose pancreática (síndrome de von Hippel-Lindau), a metástase cística e o cisto hidático.

As neoplasias císticas são lesões que, por definição, não se comunicam com o ducto pancreático principal e apresentam revestimento epitelial característico. Estas lesões constituem cerca de $1 \%$ de todas as neoplasias pancreáticas ${ }^{(3)}$ e incluem principalmente os cistadenomas mucinosos e os cistadenomas serosos, representando, respectivamente, $45 \%$ e $32 \%$ das lesões císticas do pâncreas. Embora não seja considerada uma lesão cística propriamente dita, por apresentar imagem semelhante à 
esta, a neoplasia intraductal mucinosa papilífera é estudada nesta categoria.

O aprimoramento tecnológico contínuo dos métodos de imagem como a ultra-sonografia transabdominal (UST), a tomografia computadorizada (TC), a TC helicoidal (TCH), a colangiopancreatografia por ressonância magnética (CPRM) e a ecoendoscopia (EE) têm permitido identificar, numa fase subclínica e de forma mais freqüente, lesões pancreáticas pequenas, quando a maioria dos pacientes é assintomática e não relata história prévia ou presente de doença pancreática.

Apesar da evolução tecnológica, existem lesões que apresentam imagens ultrasonográficas convencionais e tomográficas com aspectos variáveis, sendo, muitas vezes, difícil a definição diagnóstica. O cistadenoma seroso unilocular macrocístico tem imagem radiológica semelhante ao pseudocisto e ao cistadenoma mucinoso, constituindo um dos exemplos de situações clínicas difíceis para o diagnóstico diferencial exclusivamente por método de imagem.

Este artigo tem por objetivo fazer uma revisão sobre as principais neoplasias císticas primárias do pâncreas, com ênfase na aplicação da EE no diagnóstico definitivo destas lesões. As neoplasias intraductais mucinosas papilíferas, por seu aspecto muito semelhante às lesões císticas, também são descritas.

\section{ECOENDOSCOPIA - NOÇÕES BÁSICAS}

A EE é o método de investigação que combina a endoscopia digestiva ao ultrasom e produz, por meio de um transdutor de alta freqüência situado na extremidade do endoscópio, imagens de alta resolução. Ela é classificada, segundo o método de escaneamento utilizado, em radial ou linear, e segundo a indicação clínica, em diagnóstica ou terapêutica. As indicações clínicas diagnósticas são divididas em três categorias principais: anormalidades subepiteliais (também conhecidas como "submucosas"), estadiamento de neoplasias e avaliação de doenças biliopancreáticas. As indicações terapêuticas incluem, entre outros, procedimentos como a drenagem de pseudocisto pancreático e a neurólise do tronco celíaco (para a dor de origem neoplásica).
O pâncreas é bem visualizado por ambas as técnicas, radial ou linear, devido à situação anatômica e pela aposição direta do transdutor na parede gástrica e duodenal imediatamente acima ou ao lado dele. A EE permite o estudo detalhado do parênquima e ducto pancreáticos em toda a extensão da glândula, sem a interposição de gás ou gordura abdominal. Existe boa evidência científica de que a EE pode identificar lesões não detectadas anteriormente e, com isso, esclarecer alterações identificadas por outros métodos de imagem como a TC e a RM, sendo hoje, uma vez descartada previamente a presença de doença a distância, considerada padrão-ouro para avaliação deste órgão.

A técnica linear, também conhecida como setorial, possibilita a realização de procedimentos complementares invasivos e, entre estes, a punção por agulha fina guiada em tempo real (EE-PAAF), a qual permite a aquisição de material líquido, celular ou tecidual para análise laboratorial e citológica. Tanto a EE diagnóstica quanto os procedimentos intervencionistas terapêuticos são considerados métodos segu$\operatorname{ros}^{(\mathbf{4 , 5})}$, com taxas altas de sensibilidade, especificidade e eficácia, respectivamente, de $92 \%, 93 \%$ e $92 \%{ }^{(5)}$. Em todas as situações clínicas mencionadas, a EE possibilita o fornecimento de informações relevantes que "pesam" na decisão terapêutica dos pacientes. Como vantagens, a EE-PAAF permite, de forma direta e com menor risco de complicação, o diagnóstico de lesões infracentimétricas e minimiza o risco de disseminação de células malignas, uma vez que o trajeto da agulha normalmente está incluído no procedimento de ressecção, caso esta seja a indicação terapêutica do paciente. A EE-PAAF está contra-indicada quando a informação fornecida por ela não contribui para a decisão terapêutica do paciente e quando vasos sanguíneos são visualizados interpostos no trajeto de punção. O distúrbio de coagulação é contraindicação relativa, pois o trauma secundário à técnica de punção é pequeno, sendo esta, portanto, uma das alternativas para diagnóstico neste grupo de pacientes.

De modo geral, as complicações associadas aos procedimentos de EE intervencionista incidem em $0,3 \%$ a $2,0 \%{ }^{(6)}$. Nos procedimentos de EE-PAAF relacionados às lesões císticas pancreáticas, esta taxa pode chegar a $14 \%$, sendo esta justificada pelo tipo de lesão que, por sua vez, oferece ambiente mais propenso à complicação local (por exemplo: sangramento intracístico pós-punção). As principais complicações descritas são perfuração, que é secundária à manipulação endoscópica propriamente dita e é operador-dependente; sangramento, que é minimizado pela aplicação do sinal Doppler no trajeto projetado de punção; infecção secundária à passagem pela luz gastroduodenal, prevenida ou minimizada pela administração de antibiótico em esquema profilático (por exemplo: cefalotina, $2 \mathrm{~g}$ endovenosos uma hora antes da punção); pancreatite secundária ao trauma parenquimatoso, tratada na maioria dos casos com medidas conservadoras.

\section{AVALIAÇÃO ECOENDOSCÓPICA DO PÂNCREAS}

$\mathrm{Na}$ avaliação ecoendoscópica das lesões císticas do pâncreas devemos responder inicialmente três perguntas:

1. A lesão é intrapancreática?

2. A lesão é um pseudocisto ou uma neoplasia cística?

3. A lesão é benigna, maligna ou potencialmente maligna?

Os pseudocistos são lesões secundárias à pancreatite aguda ou crônica. Podem ser de retenção, no caso de pancreatite crônica, ou surgir como elemento a montante de uma lesão tumoral subclínica ou ainda ser necróticos, pós-evento de pancreatite aguda. À parte, existem os cistos distróficos que ocorrem em pâncreas ectópico duodenal e correspondem aos remanescentes císticos decorrentes de fenômenos inflamatórios incomuns (5\%) nas pancreatites crônicas alcoólicas ${ }^{(7)}$.

A diferenciação entre cistos benignos, malignos e pré-malignos é importante porque altera o tratamento destas lesões. Lesões benignas assintomáticas não requerem tratamento, enquanto lesões malignas ou de potencial maligno, na ausência de contra-indicação clínica, devem ser ressecadas cirurgicamente. Tomando a imagem como foco, Ahmad et al. ${ }^{(\mathbf{8})}$ demonstraram baixa taxa de concordância $(\kappa=0,24)$ para o diagnóstico diferencial entre lesões neoplásicas e não-neoplásicas entre oito ecoendosco- 
pistas experientes quando a compararam com o resultado histopatológico posterior da peça cirúrgica. Catalano et al.$^{(\mathbf{9})}$ ressaltaram que nenhuma característica ecoendoscópica isolada distinguiu a lesão benigna da maligna em 102 pacientes estudados. Dessa forma, apesar da EE ser destacada como elemento valioso para estas respostas, o diagnóstico com base apenas na interpretação das imagens adquiridas apresenta resultados controversos.

O diagnóstico diferencial das lesões císticas do pâncreas com base apenas na interpretação da imagem radiológica é, portanto, possível. Mas, a semelhança de algumas de suas imagens e mesmo a presença de elementos morfológicos incomuns o tornam difícil, a exemplo do que se observa no cistadenoma seroso macrocístico.

\section{AVALIAÇÃO CITOLÓGICA E LABORATORIAL DO PÂNCREAS}

O esclarecimento pelo diagnóstico citológico pode ser feito por meio da colheita de material pela punção por EE e análise citológica. $\mathrm{O}$ volume de material líquido para análise depende do tamanho destas lesões. Portanto, o volume pequeno pode constituir um fator limitante para o diagnóstico definitivo da lesão. Por outro lado, a presença de células glicogênio-positivo e a ausência de mucina são diagnósticos de cistadenoma seroso de pâncreas ${ }^{\mathbf{1 0})}$.

Neste ponto, a EE-PAAF tem sido considerada como etapa representativa na direção do diagnóstico citológico da lesão, uma vez que apresenta morbidade e taxa de complicação baixas.

O material recolhido pela EE-PAAF líquido e microbiópsia - é preparado de forma convencional para análise laboratorial e citológica. A análise laboratorial do líquido pancreático inclui a dosagem de amilase, lipase, viscosidade líquida, de diferentes marcadores tumorais (CEA, CA19.9, CA72.4, CA15.3, mucina M1), e mais recentemente, a pesquisa de Ki-ras. A análise destes fluidos tem sido objeto de interesse crescente para estudos e parece desempenhar papel na decisão terapêutica destes pacientes, colaborando para o diagnóstico diferencial entre lesões com diagnóstico citológico duvidoso e potencial maligno indefinido. No entanto, seu valor prático ainda requer mais estudos e confirmação, embora já exista tabela com valores de corte para os diferentes tipos de lesões císticas (Tabela 1) ${ }^{(\mathbf{1 1})}$. Catalano et al. ${ }^{(\mathbf{9})}$ demonstraram que o diagnóstico diferencial de neoplasias císticas pancreáticas foi fortalecido pela análise do conteúdo líquido do cisto. Tomando por base concentração elevada de antígeno carcinoembrionário $(\mathrm{CEA}>300 \mathrm{ng} / \mathrm{ml}$ ) e da viscosidade líquida $(>1,6)$, os autores foram capazes de distinguir eficazmente a neoplasia cística mucinosa da serosa e do pseudocisto inflamatório em 102 pacientes. O CEA acima de 1.200 permitiu a distinção das lesões mucinosas não-malignizadas dos cistadenocarcinomas. Em um outro estudo ${ }^{(\mathbf{1 2})}$, a associação da análise citológica com os marcadores tumorais (CEA > 400 $\mathrm{ng} / \mathrm{ml}$ e Ca19.9 > 50.000 U/ml) identificou $80 \%$ dos casos de lesões mucinosas.

Porém, mesmo reconhecendo que a EE e a EE-PAAF representam avanços reais para o estudo das lesões pancreáticas, ainda cerca de um terço das lesões císticas permanece sem o diagnóstico definido. Técnicas atuais de investigação, como a citologia e a determinação das taxas de amilase, lipase e marcadores tumorais, eventualmente, ainda não são suficientemente discriminativos. Nestes casos, a análise genética e a expressão protéica dos elementos que compõem estas lesões podem trazer informações adicionais e, por conseguinte, representar um avanço a mais.

\section{NEOPLASIA CÍSTICA SEROSA}

Os tumores pancreáticos císticos serosos são neoplasias císticas compostas por células epiteliais cuboidais benignas e representam cerca de $1 \%$ a $2 \%$ dos tumores exócrinos pancreáticos ${ }^{(\mathbf{1 3})}$. A etiologia e a patogênese são desconhecidas e é rara a transformação maligna destas lesões. O seu diagnóstico é feito em torno da sétima década de vida, em geral secundário à investigação de sintomas pouco específicos como a epigastralgia, ou pela origem de sintomas compressivos abdominais ${ }^{(7)}$, e acometem mais freqüentemente as mulheres que os homens (3:2). Localizam-se predominantemente na cabeça pancreática (40\%), medem em torno de 15 a $20 \mathrm{~cm} \mathrm{no}$ momento do diagnóstico e são ricamente vascularizadas. Pela baixa probabilidade de transformação maligna, até hoje apenas oito casos foram relatados na literatura, e seu tratamento consiste no acompanhamento clínico ${ }^{(\mathbf{1 4 , 1 5})}$. A cirurgia é reservada para os casos sintomáticos, sendo, portanto, o prognóstico dos pacientes excelente.

Morfologicamente, os cistadenomas serosos são caracteristicamente microcísticos e multiloculares (Figura 1). O aspecto ecográfico típico é de massa tumoral hipoecóica, heterogênea, com limites bem definidos, composta por vários compartimentos de tamanho milimétrico com conteúdo anecóico, sem "débris" e com reforço posterior. Os compartimentos são separados por septos finos e regulares, o que dá à lesão um aspecto global tipo "colméia". $\mathrm{A}$ EE é muito útil no estudo destas lesões por seu alto poder de resolução, sendo possível demonstrá-las detalhadamente. Eventualmente, podem apresentar-se como lesões macrocísticas e raramente apresentam estruturas internas. Em cerca de $30 \%$ dos casos apresentam uma porção

Tabela 1 Reavaliação do valor dos marcadores tumorais e bioquímicos no líquido pancreático, no diagnóstico de 130 lesões císticas do pâncreas estudadas no Hospital Beaujon (cistadenomas serosos: $n=24$; cistadenomas mucinosos e cistadenocarcinomas: $n=36$; pseudocistos pancreáticos: $n=70)^{(\mathbf{1 2})}$.

\begin{tabular}{|l|l|l|l|l|l|l|}
\hline Marcador & \multicolumn{1}{|c|}{ Valor limite } & Diagnóstico & \multicolumn{1}{|c|}{ S } & \multicolumn{1}{|c|}{$\mathrm{E}$} & \multicolumn{1}{|c|}{ VPP } & VPN \\
\hline Amilase & $>5.000 \mathrm{U} / \mathrm{l}$ & PSC & $93 *(94)$ & $82(74)$ & $86(85)$ & $91(98)$ \\
Ca19.9 & $>50.000 \mathrm{U} / \mathrm{ml}$ & CM/C-adeno & $72(75)$ & $84(90)$ & $63(67)$ & $88(90)$ \\
CEA & $>400 \mathrm{ng} / \mathrm{ml}$ & CM/C-adeno & $57(50)$ & $99(100)$ & $96(100)$ & $85(85)$ \\
CEA & $<5 \mathrm{ng} / \mathrm{ml}$ & CS & $92(100)$ & $87(86)$ & $61(54)$ & $98(100)$ \\
Ca72.4 & $>40 \mathrm{U} / \mathrm{ml}$ & CM/C-adeno & $73(63)$ & $99(98)$ & $96(95)$ & $84(85)$ \\
Mucina M1 & $>1.200 \mathrm{U} / \mathrm{ml}$ & CM/C-adeno & $41(30)$ & $93(100)$ & $71(100)$ & $79(79)$ \\
\hline
\end{tabular}

Valores entre parênteses foram publicados previamente por nosso grupo. * Grau de exatidão dos marcadores (números são porcentagens). S, sensibilidade; E, especificidade; VPP, valor preditivo positivo; VPN, valor preditivo negativo; CEA, antígeno carcinoembrionário; PSC, pseudocistos pancreáticos; CM, cistadenomas mucinosos; C-adeno, cistadenocarcinomas; CS, cistadenomas serosos. 
central de fibrose ou calcificação, descrita como "cicatriz central" (7,16). Em geral, o ducto pancreático principal não está envolvido no processo ou está obstruído pela lesão serosa. Assim, três formas são descritas por ordem decrescente de freqüência: o cistadenoma microcístico, a forma mista (micro e macrocistos) e o cistadenoma macrocístico (os cistos medem em geral mais que $2 \mathrm{~cm}$ cada).

A TC deve ser realizada como método complementar e contribui para o diagnóstico das lesões macrocísticas e mistas, ao contrário das lesões macrocísticas, cujas imagens não são específicas. A lesão microcística, antes da injeção do meio de contraste, se apresenta como lesão hipodensa bem limitada com área de calcificação central, já descrita anteriormente. Após a infusão, ocorre exacerbação dos septos finos, relembrando o aspecto tipo colméia mencionada acima. Embora tomando apenas por base a interpretação da imagem radiológica o diagnóstico diferencial das lesões císticas do pâncreas seja possível, a semelhança entre algumas das imagens adquiridas e mesmo a presença de elementos morfológicos incomuns o tornam difícil, a exemplo do que se observa no cistadenoma seroso macrocístico.

Neste caso ou quando há dúvida diagnóstica, a EE-PAAF está indicada, pois permite o diagnóstico citológico. Estas lesões são circundadas por epitélio estratificado, rico em glicogênio (Figura 2). O volume do aspirado é fator importante para a sua qualificação e depende do tamanho da lesão. Portanto, volume pequeno pode ser um fator limitante para o seu diagnóstico definitivo. Por outro lado, a presença de células glicogênio-positivas e a ausência de mucina são diagnósticos de cistadenoma seroso de pâncreas ${ }^{(\mathbf{1 0})}$. A presença de mucina ou "débris" no interior destas lesões é extremamente incomum, e uma vez diagnosticada deve levar à suspeita de cistadenoma mucinoso e não seroso.

\section{NEOPLASIA CÍSTICA MUCINOSA}

Os tumores císticos mucinosos do pâncreas são neoplasias epiteliais císticas compostas por células colunares produtoras de mucina circundadas por estroma tipo ovariano e que não se comunicam com o ducto pancreático principal. As neoplasias císticas pancreáticas, hepáticas e do retroperitônio são semelhantes. Na etiologia destas lesões considera-se possível a derivação do componente estromal primordial ovariano, argumento fundamentado, entre outros, na morfologia, tendência à luteinização e na presença de células tipo-hilares observadas nestas lesões. A hipótese atual é de que o estroma ovariano ectópico incorporado no pâncreas durante a embriogênese pode produzir hormônios e fatores de crescimento induzindo o epitélio circunjacente a proliferar e formar tumores císticos ${ }^{(17)}$. Considerando que a gônada primordial esquerda e o pâncreas dorsal estão lado a lado durante a quarta e quinta semanas do desenvolvimento intra-uterino, esta hipótese poderia explicar a predileção dessas lesões pelo segmento corpo-caudal do pâncreas (70\% a 90\%).

Acometem mais freqüentemente as mulheres que os homens (6:1), na faixa etária entre 40 e 50 anos. De acordo com o grau de displasia epitelial são classifica- das como adenoma, "borderline" (displasia de baixo grau) ou carcinoma não-invasivo ou invasivo. Embora mais de 500 casos já tenham sido registrados na literatura, são considerados como lesões raras, representando cerca de $2 \%$ a $5 \%$ dos tumores pancreáticos exócrinos ${ }^{(\mathbf{1 8})}$. Seu tamanho é muito variável, podendo medir de 1 a $20 \mathrm{~cm}$, sendo as lesões menores que 3 $\mathrm{cm}$ mais frequientemente benignas e as maiores que $8 \mathrm{~cm}$, em geral, malignas. Portanto, o correto diagnóstico dessas lesões é fundamental, uma vez que o prognóstico está diretamente relacionado com o grau de atipia celular, sendo excelente para os tumores não-invasivos completamente ressecados ${ }^{(17)}$.

Morfologicamente, os cistadenomas mucinosos são caracteristicamente macrocísticos, sendo raramente microcístico, uni ou multiloculares (Figura 3). Em geral, estas lesões são uniloculares, anecóicas com conteúdo líquido espesso e "débris", as paredes são relativamente finas e bem individualizadas do parênquima pancreático e freqüentemente existem áreas internas de espessamento parietal que, às vezes, formam projeções intraluminares. A presença de espessamento ou irregularidade da parede do cisto deve levar à suspeição de malignidade ${ }^{(\mathbf{1 9})}$. Pode haver, em $15 \%$ dos casos, áreas de calcificações parietais, as quais são visualizadas como estruturas hiperecogênicas arciformes ${ }^{(7)}$.

A TC mostra lesão hipodensa bem limitada, em geral arredondada, ressaltando as calcificações periféricas, quando presentes. Por outro lado, antes da injeção do meio de contraste e especialmente nas lesões pequenas, não é visível o conteúdo viscoso

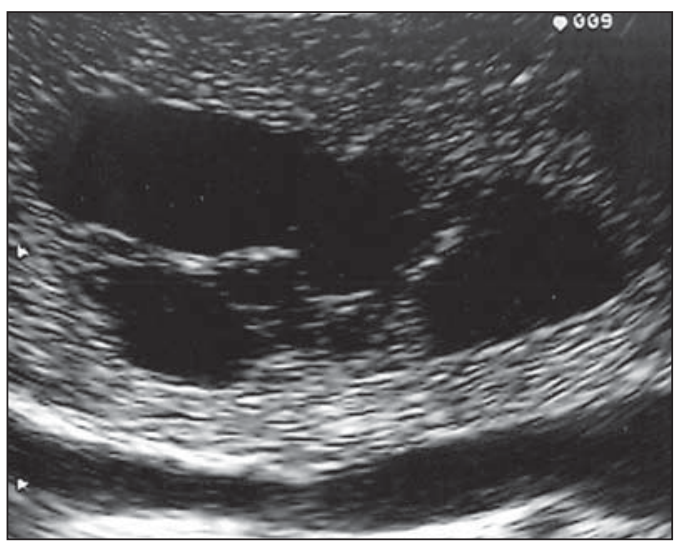

1

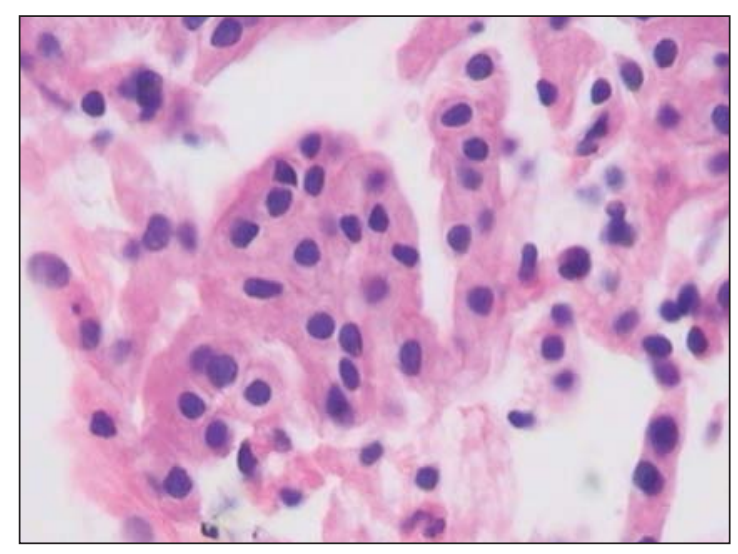

2

Figura 1. Ecoendoscopia. Cistadenoma seroso situado na cabeça pancreática com aspecto multilocular constituído por formações císticas de tamanho variável, sem comunicação com o ducto pancreático principal. Lesão com $28,7 \times 16,7 \mathrm{~mm}$.

Figura 2. Citologia. Presença de células cuboidais ricas em glicogênio representativas de neoplasia cística serosa. Hematoxilina-eosina, $400 \times$. 
com "débris". Entretanto, as projeções intraluminares são facilmente visualizadas nas lesões de maior tamanho ${ }^{(7)}$.

O diagnóstico diferencial dessas lesões é importante e, por ordem de freqüência, consideramos primeiro o pseudocisto pancreático. O erro diagnóstico neste caso leva à realização inadequada de derivações cistojejunais, drenagem percutânea ou acompanhamento clínico. Pode, ainda, um cistadenoma seroso macrocístico ser confundido como mucinoso e ser ressecado sem indicação clínica. A presença de células inflamatórias circunjacentes pode complicar ainda mais o diagnóstico correto dessas lesões ${ }^{(7)}$. Portanto, estas são algumas das razões que explicam a realização da EEPAAF para o diagnóstico correto delas.

A punção dos cistadenomas guiada em tempo real por ecoendoscopia é relativamente simples e segura. A agulha é posicionada no centro da lesão e o conteúdo líquido é completamente aspirado. Porém, cuidadoso exame do parênquima circunjacente é necessário para a exclusão de lesões a jusante e concomitantes à formação cística. Quando existem áreas de espessamento ou áreas sólidas hipoecóicas, estas devem ser incluídas nos movimentos da punção, pela maior probabilidade de haver células informativas nesta região, aumentando assim a qualidade celular do aspirado. O material líquido contido nestas lesões é em geral viscoso, o que pode representar um problema no momento da aspiração, pois a agulha pode se tornar obstruída ou o tempo para aspirar toda a lesão pode ser longo. Neste caso, o uso de agulha com calibre maior $(19 \mathrm{G})$ pode ajudar na colheita do material. Sangramento intracístico é incomum, principalmente se a lesão for previamente estudada pelo sinal Doppler. O esvaziamento completo da lesão colabora para a prevenção da infecção do cistadenoma como resultado da EEPAAF e sempre deve ser tentado. Por outro lado, a repetição da punção pode aumentar o risco de complicações.

A avaliação citológica consiste na procura de células epiteliais colunares produtoras de mucina e da própria mucina (Figura 4). Para melhorar a qualidade do material aspirado, é importante evitar ao máximo a contaminação do material com células gástricas ou duodenais no aspirado no momento da passagem da agulha pela parede gástrica ou duodenal. A presença de células gástricas e/ou duodenais pode resultar em citologia falso-positiva para células mucinosas. Sperti et al. ${ }^{(20)}$, estudando a utilidade da análise sérica e intracística das enzimas e marcadores tumorais, observaram que a citologia desse material apresentou taxas de sensibilidade e especificidade de $48 \%$ e $100 \%$, respectivamente. Quando a lesão se trata de cistadenocarcinoma, as células epiteliais malignas são visualizadas mais facilmente, particularmente quando existe uma lesão sólida no interior da lesão cística e esta foi traspassada pela agulha de punção ${ }^{(21)}$.

\section{NEOPLASIA INTRADUCTAL MUCINOSA PAPILÍFERA}

A neoplasia intraductal mucinosa papilífera, antes confundida com as neoplasias císticas mucinosas, origina-se no ducto pancreático principal ou nos seus ramos principais e forma projeções papiliformes que crescem em direção à luz ductal, levando à dilatação intraductal pseudocística, uma vez que não é lesão propriamente cística. O termo neoplasia intraductal mucinosa papilífera foi proposto por Warshaw et al. em 1997, quando estes demonstraram que a ectasia ductal mucinosa e a neoplasia intraductal mucinosa papilífera eram nomes diferentes para a mesma entida$\mathrm{de}^{(22)}$. O componente epitelial papilífero, o grau de produção de mucina, da dilatação ductal cística e da invasão locorregional é variável. Outros nomes para a mesma lesão incluem papilomatose do pâncreas, adenomatose dos canais pancreáticos, doença de Itaï ou tumor ductoectático produtor de mucina, tumor mucinoso intraductal, ectasia ductal mucinosa, tumor intraductal hipersecretor de mucina, e o mais inadequado de todos, cistadenocarcinoma intraductal, que não deve ser usado.

A baixa incidência e a identificação imprecisa das neoplasias intraductais mucinosas papilíferas têm mascarado o reconhecimento dos fatores etiológicos. Fukushima e Mukai descrevem que a maioria de seus pacientes era fumante ${ }^{(23)}$. No entanto, não existe associação consistente destas lesões com outras lesões neoplásicas pancreáticas $^{(\mathbf{1 7})}$.

Até o momento, a incidência das neoplasias intraductais mucinosas papilíferas é estimada em 1\% a 3\% das neoplasias exócrinas pancreáticas, ou menor que 1 a cada 100.000 habitantes/ano ${ }^{(\mathbf{1 3})}$. A maioria destas lesões ocorre no ducto pancreático principal e seus ramos na cabeça pancreática $(60 \% \text { a } 70 \%)^{(\mathbf{7 , 2 4 , 2 5})}$. A origem multicêntrica é suspeitada pela recorrência na glândula pancreática remanescente

Figura 3. Ecoendoscopia Lesão anecóica uniloculada localizada em cabeça do pâncreas com forma alongada e contorno definido, mas irregular, medindo 32,2 $\times 7,0 \mathrm{~mm}$.

Figura 4. Citologia. Presença de células epiteliais colunares produtoras de mucina compatível com neoplasia cística mucinosa. Hematoxilina-eosina, $80 \times$.

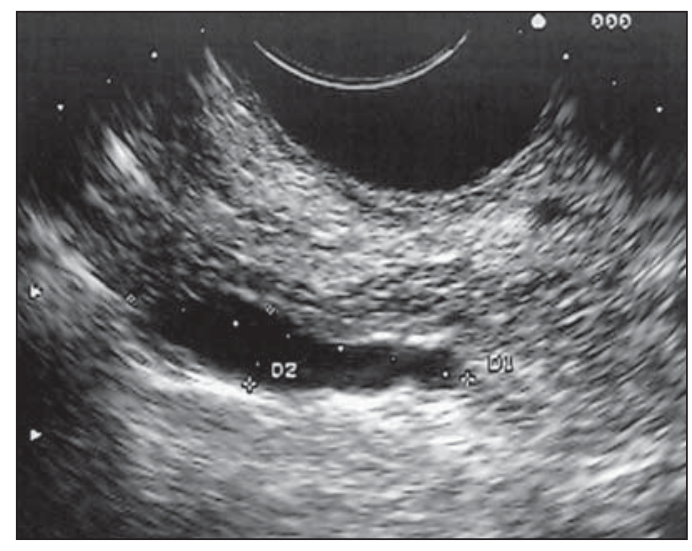

3

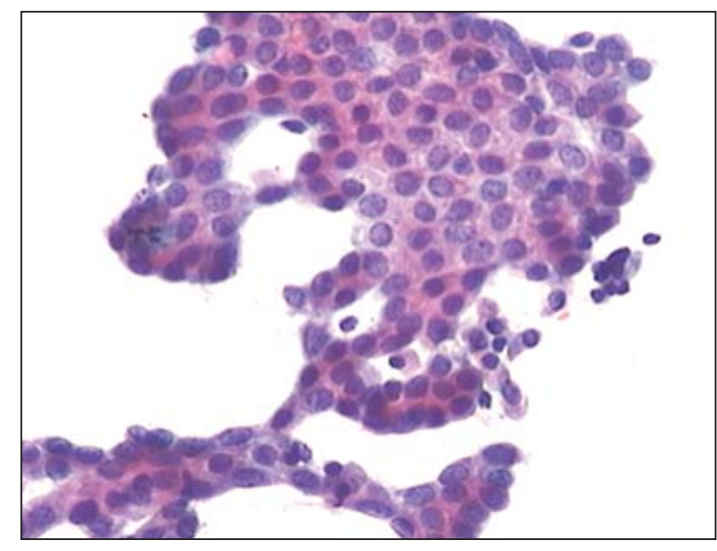

4 
após a cirurgia ${ }^{(26)}$. População com faixa etária ampla (30 a 94 anos) pode apresentar neoplasia intraductal mucinosa papilífera, entretanto, a idade média para o diagnóstico está entre a sexta e sétima décadas de vida ${ }^{(27)}$. Homens são mais acometidos que mulheres $(3: 1)^{(\mathbf{7 , 2 8})}$. Estas lesões são classificadas em benignas, "borderline" e malignas não-invasivas ou invasivas. Embora estejam sendo mais descritas, sua incidência, ainda desconhecida, é atribuída ao avanço tecnológico, ao progresso no seu reconhecimento e à recente padronização para sua classificação.

A apresentação clínica destas lesões é variável, podendo ser o paciente assintomático ou oligossintomático e ter o diagnóstico feito incidentalmente pelo achado de dilatação do ducto pancreático por outro método de imagem, ou apresentar sintomas objetivos como dor epigástrica, perda de peso, diabetes e icterícia ${ }^{(29-31)}$. No entanto, a apresentação inicial mais comum é o quadro de pancreatite aguda de origem indeterminada $(40 \%)^{(7)}$. Com o passar dos anos, o diagnóstico é mais freqüente pela recorrência dos eventos de pancreatites, que são de intensidade leve a moderada, raramente graves. As taxas de amilase e lipase sanguíneas estão freqüentemente elevadas. A EE e a colangiopancretografia endoscópica retrógrada podem ajudar no diagnóstico pré-operatório. A biópsia endoscópica ou EE-PAAF permitem o diagnóstico citológico, entretanto, é fundamental a avaliação de toda a peça cirúrgica após a ressecção para a determinação do grau de displasia de toda a lesão. Estas lesões são potencialmente malignas. Palazzo et al. relatam achados de $40 \%, 10 \%$ a $20 \%$, e $30 \%$ a $40 \%$ de lesões invasivas, displasia grave (carcinoma in situ) e hiperplasia ou displasia mínima a moderada, respectivamente ${ }^{(7)}$. O estudo dos marcadores tumorais CEA e Ca19.9 são insensíveis ${ }^{(28)}$.

As neoplasias intraductais mucinosas papilíferas têm sido confundidas com os cistadenomas mucinosos porque ambos apresentam aspecto cístico e têm componente celular semelhante. Todavia, são entidades diferentes e podem ser individualizadas facilmente porque, ao contrário das neoplasias intraductais mucinosas papilíferas, os cistadenomas mucinosos ocorrem tipicamente em mulheres, na quinta ou sexta décadas de vida, localizam-se predominantemente no corpo ou cauda pancreáticos, apresentam parede espessa e não se comunicam com o ducto pancreático.

Radiologicamente são descritas três formas: o tipo ducto principal, o tipo ducto secundário (o mais frequente, $40 \%$ ) e o tipo misto, que compromete tanto o ducto principal quanto o ducto secundário. $\mathrm{Na}$ UST e na TC estas lesões apresentam aspecto típico: lesão cística uni ou multiloculada, localizada em geral na cabeça pancreática, com contorno nítido e associada a dilatação do canal pancreático principal. A colangiopancreatografia endoscópica retrógrada tem sido considerada o exame de referência para a definição diagnóstica. $\mathrm{Na}$ abordagem inicial deste procedimento, a visualização da papila de Vater com orifício dilatado ( $40 \%$ a $50 \%$ ), pelo qual sai secreção mucóide, é patognomônica desta afecção ${ }^{(7)}$. A pancreatografia com falhas de enchimento com aparência tipo cística e com conteúdo móvel é considerada também preditiva desta lesão. Todavia, a conclusão diagnóstica pode ser dificultada pelas semelhanças com o pancreatograma da pancreatite crônica, o que inclui calcificações focais, dilatação ductal focal, irregularidades ductais e ductos secundários anormais. Além disso, a abundante quantidade de mucina intraductal e intracística pode impedir a visualização correta dos ductos secundários em até $12 \%$ dos pacientes ${ }^{(32,33)}$. E, embora a abordagem endoscópica convencional permita aspirar material líquido do ducto, ela não é capaz de detectar as lesões císticas ou tumorais no interior do parênquima que geralmente acompanham estas lesões ${ }^{(34)}$.

A EE apresenta resultados semelhantes aos da pancreatografia e superiores aos da UST, tendo a vantagem de associar a visualização da papila de Vater (Figura 5) com o estudo das formações císticas intraparenquimatosas e a avaliação da glândula como um todo, tornando assim possível o estudo de toda a árvore pancreática em um único procedimento. Permite também detectar a dilatação de canais secundários ou a presença de lesões focais hipoecóicas sugestivas de tumor intraparenquimatoso não diagnosticado previamente. Dessa forma, é possível, além de examinar a lesão pela imagem detalhada, puncionar diretamente

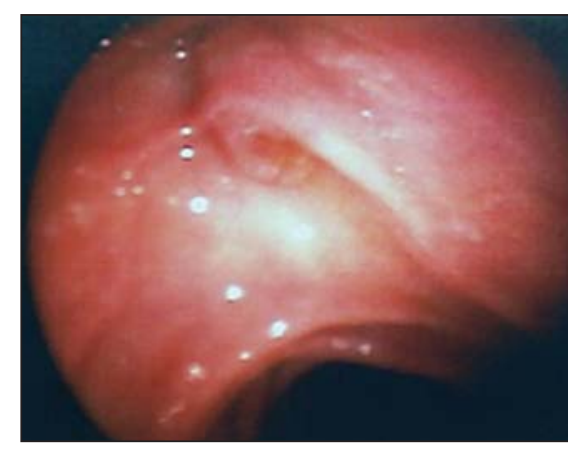

Figura 5. Papila de Vater com orifício aberto e por onde é visualizada secreção mucóide espessa, sugerindo o diagnóstico de neoplasia intraductal mucinosa papilífera.

a lesão num mesmo procedimento para diagnóstico citológico. Portanto, na ecoendoscopia são pesquisados não só o aspecto da lesão propriamente dita, mas sua relação com os ductos pancreáticos principal e secundários. O aspecto global sugere um "cacho de uva" (Figura 6). As neoplasias intraductais mucinosas papilíferas são caracterizadas como formações tipo císticas com parede externa fina, sem septos, representando os ductos dilatados (Figura 7) contendo material espesso com "débris". Nestas lesões podem ser identificados nódulos murais ou massas sólidas hipoecóicas concomitantes (Figura 8). As lesões císticas associadas às neoplasias intraductais mucinosas papilíferas apresentam aspectos morfológicos diversos, o que eventualmente pode simular cistadenomas micro ou macrocísticos. Considerando que a principal limitação da EE no diagnóstico

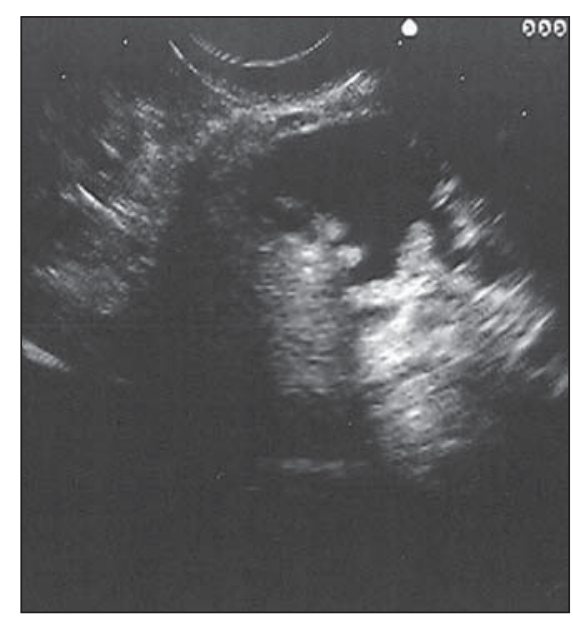

Figura 6. Ecoendoscopia: lesão cística com parede externa formando projeções intraluminares tipo "cacho de uva". 
Figura 7. Dilatação ducta da neoplasia intraductal mucinosa papilífera demonstrada detalhadamente pela ecoendoscopia.

Figura 8. Lesão com aspecto ecoendoscópico misto composta por área sólida central associada a formações císticas de tamanhos diversos. Visualiza-se a aguIha de punção, cujo trajeto atravessa a massa sólida no centro da lesão.
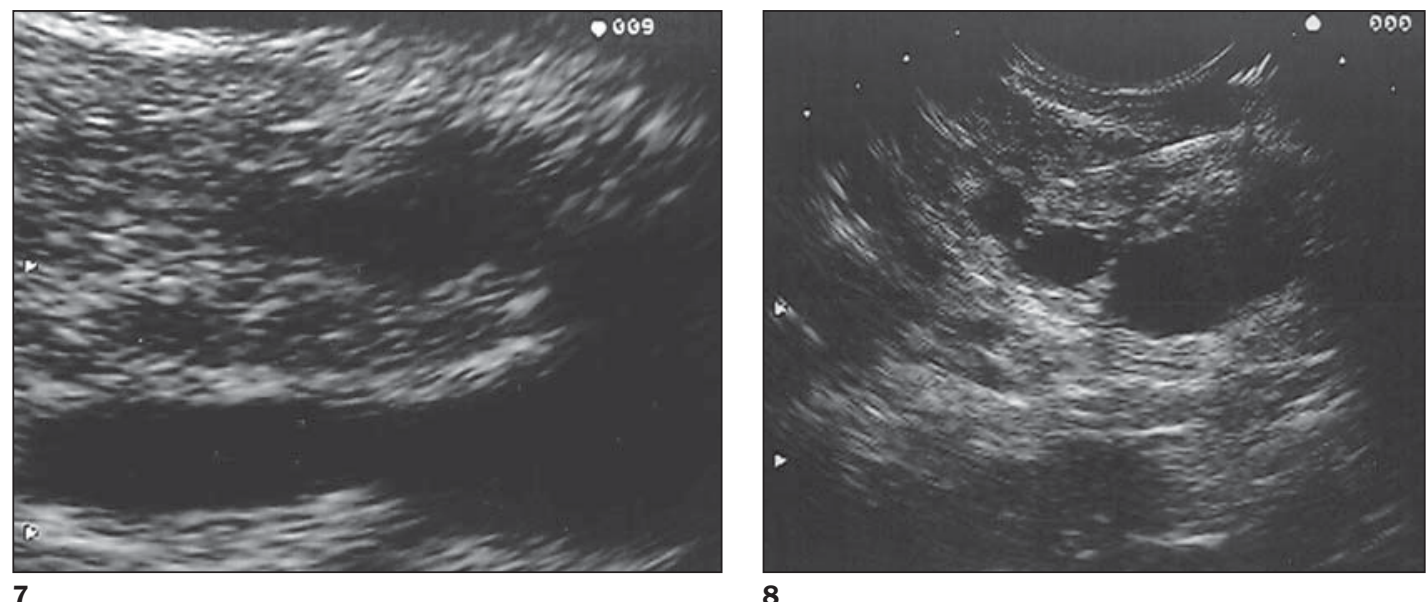

das neoplasias intraductais mucinosas papilíferas é a sua inabilidade para diferenciar entre áreas malignas de áreas inflamatórias, a aplicação da EE-PAAF tem colaborado positivamente para aumentar a especificidade dos achados ecoendoscópi$\cos ^{(35)}$. Na EE-PAAF, o volume líquido deve ser o maior possível com a finalidade de recolher material suficiente para interpretação citológica. A análise do aspirado demonstra material celular semelhante aos dos cistadenomas mucinosos.

A associação da EE com a pancreatografia retrógrada trabalha em conjunto, apresentando precisão diagnóstica pouco acima de $80 \%^{(7)}$. Na impossibilidade de se realizar a EE, a CPRM pode contribuir para o estudo dessas lesões.

Hara et $a l .{ }^{(36)}$ avaliaram a utilidade da pancreatoscopia peroral e da EE intraductal em 60 casos de neoplasias intraductais mucinosas papilíferas confirmados histologicamente. Segundo eles, a combinação das duas técnicas foi muito efetiva para o diagnóstico diferencial entre lesões benignas e malignas. Esta informação é particularmente importante na avaliação das neoplasias intraductais mucinosas papilíferas, cuja taxa de sobrevida acumulada de três anos de $95 \%$ pode ser conseguida se o manuseio do paciente for correto.

O prognóstico destas lesões é excelente para os tumores adenomatosos ou "borderline", com taxa de sobrevida de $100 \%$ em três a cinco anos. O tratamento destas lesões depende do diagnóstico da extensão longitudinal da lesão (extensão ao longo do ducto pancreático), sendo fundamental a avaliação peroperatória da lesão.

\section{OUTRAS LESÕES CÍSTICAS RARAS}

Outras entidades têm sido descritas como neoplasias císticas primárias do pâncreas, estando aí incluídos os linfangiomas císticos, os leiomiossarcomas, os cistos dermóides (teratomas) e os schwannomas. Em cada um destes grupos de lesões, pouco menos de 25 casos foram publicados até hoje. O tratamento cirúrgico é recomendado devido ao potencial maligno dessas lesões.

O linfangioma pode ocorrer em pacientes assintomáticos ou com dor abdominal. A massa apresenta aspecto uni ou multiloculado e o diagnóstico é confirmado pela EE-PAAF ou pela ressecção cirúrgica.

Os teratomas císticos são tumores extremamente raros, podem conter tecido ósseo, dente ou cabelo e causam sintomas de compressão à medida que crescem. A ressecção é recomendada ${ }^{(37)}$.

As lesões metastáticas císticas são raras e podem ser decorrentes de neoplasias malignas do pulmão, pele (melanoma), mama, ovário, cólon, estômago e tireóide. $\mathrm{Na}$ TC são identificados três padrões diferentes: massas solitárias, lesões multinodulares e comprometimento difuso da glândula sem sinal focal ${ }^{(38)}$.

\section{CONCLUSÃO}

O diagnóstico da neoplasia cística do pâncreas depende da combinação dos dados clínicos com as informações fornecidas pelos métodos de imagem. Estes, inicialmente restritos à UST, hoje incluem a TC — principalmente a TC helicoidal —, a EE e a colangiopancreatografia endoscópica retrógrada, a qual, progressivamente, vem sendo substituída pela CPRM. A tendência atual, quando não há contra-indicação técnica à sua execução (por exemplo: vaso interposto no trajeto da punção), é avaliar a lesão por meio da EE e puncioná-la com o objetivo de colher material líquido e microbiópsia para avaliação citológica e laboratorial, a fim de se aumentar as chances de se identificar, o mais precocemente possível, os pacientes com lesões potencialmente malignas, os quais se beneficiarão pelo tratamento cirúrgico.

A EE, por permitir analisar de forma detalhada a lesão cística do pâncreas, sua arquitetura interna e parede externa, se há comunicação ou não com o ducto principal, se há ou não elementos predisponentes à complicação em caso de punção (por exemplo: vasos sanguíneos interpostos) e por apresentar porcentagens altas de grau de exatidão - $82 \%$ a $91 \%, 94 \%$ a $97 \%$ e $92 \%$ a $96 \%$ para as lesões com até $2 \mathrm{~cm}, 4$ cm e $6 \mathrm{~cm}$ de tamanho, respectivamentevem sendo apontada como padrão-ouro na estratégia de identificação e no estadiamento destas lesões. A identificação de septos, nódulos murais e alterações parenquimatosas constituem os fatores preditivos mais significativos no diagnóstico das lesões císticas do pâncreas, principalmente para sua diferenciação das lesões mucinosas, apresentando taxas de sensibilidade, especificidade e grau de exatidão de $94 \%, 85 \%$ e $88 \%$, respectivamente ${ }^{(34,39)}$.

\section{REFERÊNCIAS}

1. Compagno J, Oertel JE. Mucinous cystic neoplasms of the pancreas with overt and latent malignancy 
(cystadenocarcinoma and cystadenoma). A clinicopathologic study of 41 cases. Am J Clin Pathol 1978;69:573-580.

2. Compagno J, Oertel JE. Microcystic adenomas of the pancreas (glycogen-rich cystadenomas): a clinicopathologic study of 34 cases. Am J Clin Pathol 1978;69:289-298.

3. Fernandez-del Castillo C, Warshaw AL. Cystic tumors of the pancreas. Surg Clin North Am 1995; 75: 1001-1016.

4. Bhutani MS, Hawes RH, Baron PL, et al. Endoscopic ultrasound guided fine needle aspiration of malignant pancreatic lesions. Endoscopy 1997;29: 854-858.

5. Wiersema MJ, Vilmann P, Giovannini M, Chang KJ, Wiersema LM. Endosonography-guided fineneedle aspiration biopsy: diagnostic accuracy and complication assessment. Gastroenterology 1997; 112:1087-1095.

6. Harewood GC, Wiersema MJ. Endosonographyguided fine needle aspiration biopsy in the evaluation of pancreatic masses. Am J Gastroenterol 2002;97:1386-1391.

7. Palazzo L, Hammel P, Cellier C, Ruszniewski P. Les tumeurs kystiques du pancréas. Acta Endoscopica 2000;30 Supplément 2:361-366.

8. Ahmad NA, Kochman ML, Brensinger C, et al. Interobserver agreement among endosonographers for the diagnosis of neoplastic versus non-neoplastic pancreatic cystic lesions. Gastrointest Endosc 2003;58:59-64.

9. Catalano MF, Sivak MV Jr, Rice T, Gragg LA, Van Dam J. Endosonographic features predictive of lymph node metastasis. Gastrointest Endosc 1994; 40:442-446.

10. Brugge WR. The role of EUS in the diagnosis of cystic lesions of the pancreas. Gastrointest Endosc 2000;52(6 Suppl):S18-S22.

11. Hammel P. Role of tumor markers in the diagnosis of cystic and intraductal neoplasms. Gastrointest Endosc Clin N Am 2002;12:791-801.

12. Iwase K, Takenaka H, Oshima S, et al. Determination of tumor marker levels in cystic fluid of benign liver cysts. Dig Dis Sci 1992;37:1648-1654.

13. Morohoshi T, Held G, Kloppel G. Exocrine pancreatic tumours and their histological classification. A study based on 167 autopsy and 97 surgical cases. Histopathology 1983;7:645-661.

14. Ishikawa T, Nakao A, Nomoto S, et al. Immunohistochemical and molecular biological studies of serous cystadenoma of the pancreas. Pancreas 1998;16:40-44.
15. George DH, Murphy F, Michalski R, Ulmer BG. Serous cystadenocarcinoma of the pancreas: a new entity? Am J Surg Pathol 1989;13:61-66.

16. Torresan F, Casadei R, Solmi L, Marrano D, Gandolfi L. The role of ultrasound in the differential diagnosis of serous and mucinous cystic tumours of the pancreas. Eur J Gastroenterol Hepatol 1997;9:169-172.

17. Zamboni G, Scarpa A, Bogina G, et al. Mucinous cystic tumors of the pancreas: clinicopathological features, prognosis, and relationship to other mucinous cystic tumors. Am J Surg Pathol 1999;23: 410-422.

18. Thompson LD, Becker RC, Przygodzki RM, Adair CF, Heffess CS. Mucinous cystic neoplasm (mucinous cystadenocarcinoma of low-grade malignant potential) of the pancreas: a clinicopathologic study of 130 cases. Am J Surg Pathol 1999;23:1-16.

19. Gress F, Gottlieb K, Cummings O, Sherman S, Lehman G. Endoscopic ultrasound characteristics of mucinous cystic neoplasms of the pancreas. Am J Gastroenterol 2000;95:961-965.

20. Sperti C, Pasquali C, Guolo P, Polverosi R, Liessi G, Pedrazzoli S. Serum tumor markers and cyst fluid analysis are useful for the diagnosis of pancreatic cystic tumors. Cancer 1996;78:237-243.

21. Centeno BA, Warshaw AL, Mayo-Smith W, Southern JF, Lewandrowski K. Cytologic diagnosis of pancreatic cystic lesions. A prospective study of 28 percutaneous aspirates. Acta Cytol 1997;41:972980.

22. Rivera JA, Fernandez-del Castillo C, Pins M, et al. Pancreatic mucinous ductal ectasia and intraductal papillary neoplasms. A single malignant clinicopathologic entity. Ann Surg 1997;225:637-644; discussion 644-646.

23. Fukushima N, Mukai K. Pancreatic neoplasms with abundant mucus production: emphasis on intraductal papillary-mucinous tumors and mucinous cystic tumors. Adv Anat Pathol 1999;6:65-77.

24. Azar C, Van de Stadt J, Rickaert F, et al. Intraductal papillary mucinous tumours of the pancreas. Clinical and therapeutic issues in 32 patients. Gut 1996;39:457-464.

25. Conley CR, Scheithauer BW, van Heerden JA, Weiland LH. Diffuse intraductal papillary adenocarcinoma of the pancreas. Ann Surg 1987;205: 246-249.

26. Loftus EV Jr, Olivares-Pakzad BA, Batts KP, et al. Intraductal papillary-mucinous tumors of the pancreas: clinicopathologic features, outcome, and nomenclature. Members of the Pancreas Clinic, and
Pancreatic Surgeons of Mayo Clinic. Gastroenterology 1996;110:1909-1918.

27. Paal E, Thompson LD, Frommelt RA, Przygodzki RM, Heffess CS. A clinicopathologic and immunohistochemical study of 35 anaplastic carcinomas of the pancreas with a review of the literature. Ann Diagn Pathol 2001;5:129-140.

28. Yamada M, Kozuka S, Yamao K, Nakazawa S, Naitoh Y, Tsukamoto Y. Mucin-producing tumor of the pancreas. Cancer 1991;68:159-168.

29. Yasuda H, Takada T, Amano H, Yoshida M. Surgery for mucin-producing pancreatic tumor. Hepatogastroenterology 1998;45:2009-2015.

30. Traverso LW, Peralta EA, Ryan JA Jr, Kozarek RA. Intraductal neoplasms of the pancreas. Am J Surg 1998;175:426-432.

31. Kloppel G. Clinicopathologic view of intraductal papillary-mucinous tumor of the pancreas. Hepatogastroenterology 1998;45:1981-1985.

32. Paal E, Thompson LD, Przygodzki RM, Bratthauer GL, Heffess CS. A clinicopathologic and immunohistochemical study of 22 intraductal papillary mucinous neoplasms of the pancreas, with a review of the literature. Mod Pathol 1999;12:518-528.

33. Pavone E, Mehta SN, Hilzenrat N, et al. Role of ERCP in the diagnosis of intraductal papillary mucinous neoplasms. Am J Gastroenterol 1997;92: 887-890.

34. Koito K, Namieno T, Nagakawa T, Shyonai T, Hirokawa N, Morita K. Solitary cystic tumor of the pancreas: EUS-pathologic correlation. Gastrointest Endosc 1997;45:268-276.

35. Bounds BC. Diagnosis and fine needle aspiration of intraductal papillary mucinous tumor by endoscopic ultrasound. Gastrointest Endosc Clin N Am 2002;12:735-745, vii.

36. Hara T, Yamaguchi T, Ishihara T, et al. Diagnosis and patient management of intraductal papillarymucinous tumor of the pancreas by using peroral pancreatoscopy and intraductal ultrasonography. Gastroenterology 2002;122:34-43.

37. Anderson MA, Scheiman JM. Nonmucinous cystic pancreatic neoplasms. Gastrointest Endosc Clin N Am 2002;12:769-779, viii.

38. Ferrozzi F, Bova D, Campodonico F, Chiara FD, Passari A, Bassi P. Pancreatic metastases: CT assessment. Eur Radiol 1997;7:241-245.

39. Song MH, Lee SK, Kim MH, et al. EUS in the evaluation of pancreatic cystic lesions. Gastrointest Endosc 2003;57:891-896. 